\title{
Observation on the Efficacy of Autologous Blood Transfusion Combined with Controlled Hypotension in Patients with Spinal Internal Fixation during the Outbreak of New Coronavirus Pneumonia in Western Guangxi (in Guixi)
}

\author{
Chengkua Huang1, Guosheng Su2 ${ }^{2}$, Yong Zhao', Chunxian Lu' ${ }^{1}$, Sheng Nong ${ }^{3}$, Xiaohua Huang4\# \\ ${ }^{1}$ Department of Spine and Orthopedics, Southwest Hospital Affiliated to Youjiang Medical College for Nationalities, Baise \\ People's Hospital, Baise, China \\ ${ }^{2}$ Department of Laboratory Medicine, Orthopedic Hospital of Integrated Traditional Chinese and Western Medicine in Guigang, \\ Guigang, China \\ ${ }^{3}$ School of Public Health and Management, Guangxi Baiyoujiang Medical College for Nationalities, Baise, China \\ ${ }^{4}$ Department of Laboratory Medicine, Yunyang Hospital of Traditional Chinese Medicine, Chongqing, China \\ Email: 1244943047@qq.com, ${ }^{* 563449581 @ q q . c o m,{ }^{*} 54076125 @ q q . c o m}$
}

How to cite this paper: Huang, C.K., Su, G.S., Zhao, Y., Lu, C.X., Nong, S. and Huang, X.H. (2021) Observation on the Efficacy of Autologous Blood Transfusion Combined with Controlled Hypotension in Patients with Spinal Internal Fixation during the Outbreak of New Coronavirus Pneumonia in Western Guangxi (in Guixi). Open Journal of Blood Diseases, 11, 15-23. https://doi.org/10.4236/ojbd.2021.111003

Received: January 15, 2021

Accepted: March 2, 2021

Published: March 5, 2021

Copyright $\odot 2021$ by author(s) and Scientific Research Publishing Inc. This work is licensed under the Creative Commons Attribution International License (CC BY 4.0).

http://creativecommons.org/licenses/by/4.0/

\section{Abstract}

Objective: To explore the therapeutic effect of autologous blood reinfusion combined with controlled hypotension in surgical spinal fixation during the outbreak of COVID-19. Methods: 30 patients with spinal internal fixation autologous blood transfusion combined with controlled hypotension were selected as the subjects during the epidemic period from December 2019 to June 2020 in our hospital and during the operation, on the basis of routine blood pressure reduction, the American Haemonetice Corporation autologous blood continuous reinfusion system was used to infuse the blood recovered during the operation to the patient through filtration and other procedures. Results: 30 patients had no complications such as fever and hemolysis; And after the operation, the tube was dialed according to the drainage volume, the cervical thoracic and lumbar brace was customized, and the patient walked on the ground for one week; After no abnormalities, the patient was discharged. Intraoperative comparison of white blood cells (WBC), red blood cells (RBC), red blood cell pressure (HCT), hemoglobin (HGB), and coagulation

\footnotetext{
${ }^{\star}$ Co-first author.

${ }^{*}$ Corresponding author.
} 
time (PT) of patients with autologous blood before and after transfusion showed statistically significant differences before and after surgery $(P<0.001)$. Conclusion: During the new coronavirus pneumonia epidemic, the internal fixation of spinal surgery used a recovery machine to collect intraoperative blood for reinfusion. Intraoperative antihypertensive drugs were used to control blood pressure within a certain safe range. The postoperative clinical observation effect was significant and safe; especially at present the clinical significance during the epidemic was significant.

\section{Keywords}

New Coronavirus Pneumonia, Spinal Internal Fixation Surgery Autologous Blood Reinfusion, Controlled Hypotension, Curative Effect

\section{Introduction}

With the development of society and the advancement of science and technology, the incidence of trauma continues to rise, and high-energy trauma is becoming more common. From 2004 to 2015, accidents on highways and buildings in China involved more than 200,000 casualties, with a total fatality rate of $33.87 \%$. Beginning in the 1970s, the construction of trauma centers in developed countries has replaced the three-peak model by the single-peak model [1]. According to the fifth general survey of health services in China, about 700,000 people die from trauma every year, ranking the fifth of the total cause of death [2]. Trauma will cause great pain and casualties in a very short period of time, and the losses caused by subsequent injuries and disabilities are more serious. The annual medical expenses caused by trauma in China are as high as 65 billion yuan [2] [3]. In my country, according to statistics, the amount of clinical blood used is increasing at a rate of $10 \%$ per year. The blood supply is in short supply. Patients undergoing surgery must donate blood through mutual assistance before surgery. In addition, some relatives of patients cannot donate blood due to blood diseases; especially patients undergoing emergency surgery during the COVID-19 epidemic have seriously affected the lives and treatment capabilities of patients [4]. How to safely and effectively manage blood for these special groups of people during orthopedic surgery during the epidemic is worth pondering. Wu Wei et al. [5] reached an expert consensus on the standardized procedures for orthopedic diagnosis and treatment during the new coronavirus pneumonia epidemic. Zhang Yaoshen, Lv Hongzhi, Wang Xiuli et al. [6] [7] [8] provided us with a reference plan for orthopedic surgery protection strategies, factors affecting fracture site changes, and anesthesia options under the new coronavirus pneumonia epidemic. West Guangxi (Baise area) is located at the junction of Yunnan, Guangxi and Guizhou, with remote location, steep terrain, and complicated terrain. With economic development and accelerated trade, traumatic incidents frequently occur due to traffic, real estate construction, and leisure and entertainment. In 
addition, during the new coronavirus pneumonia epidemic, the occurrence of these events has put blood stations and medical and health systems facing severe tests. Timely and effective blood transfusion and hemostatic measures are related to the treatment of the lives of the wounded. In recent years, blood management is an emerging discipline and a new research focus. Intraoperative and postoperative autologous blood transfusion is more widely used clinically, especially during the epidemic period, and many advantages are more prominent. In 2017, our hospital introduced the American autologous blood reinfusion system. Our department began to apply the American Haemonetice Corporation autologous blood reinfusion system to autologous blood reinfusion for patients with spinal surgery. Good results have been achieved without adverse reactions. Autologous blood transfusion is safe and effective during the outbreak of new coronavirus pneumonia.

\section{Materials and Methods}

\subsection{General Information}

This group selected 30 patients with spinal internal fixation who were hospitalized for surgery from December 2019 to June 2020 during the epidemic. Requirements for selected patients: A. The operation is more difficult, the operation time is estimated to be long, and there is a lot of blood during the operation; B. Those who have a history of severe blood transfusion reactions; C. Previous blood matching difficulties or rare blood types; D. Strengthen epidemiological screening of inpatients; E. All patients and their companions will be tested for new coronavirus nucleic acid and chest CT. Among the patients, 3 were cervical fractures, 10 were thoracolumbar fractures, 3 were multilevel cervical spinal stenosis, 11 were lumbar spinal stenosis, and 3 were lumbar unstable spondylolisthesis, see Table 1 below for details. Including 20 men and 10 women, aged 15 - 70 years. The average is 51 years old. During the operation and after the operation, the Haemonetice Corporation autologous blood reinfusion system of the United States was used to return the blood recovered during the operation to the patient.

\subsection{Intraoperative Autotransfusion Method}

In this study, the intraoperative autologous blood reinfusion system of Haemonetice Corporation of the United States was used to connect a sterile tube before the operation, and put normal saline moistened with heparin into the blood reservoir. Use a suction device to fully absorb the blood during the operation,

Table 1. Basic information of 30 subjects.

\begin{tabular}{cc}
\hline Patient condition & Cases \\
\hline cervical fractures & 3 \\
thoracolumbar fractures & 10 \\
multilevel cervical spinal stenosis & 3 \\
lumbar spinal stenosis & 11 \\
lumbar unstable spondylolisthesis & 3 \\
\hline
\end{tabular}


including bloody gauze, square yarn, etc. The concentrated red blood cells are collected in the blood transfusion bag through filtration, purification, washing, etc., and then returned to the patient during the operation.

\subsection{The Statistical Processing}

The statistical processing uses the SPSS 20.0 software to carry on the statistical analysis to the obtained data. Measurement data is expressed by $(\mathrm{X} \pm \mathrm{s})$, and comparison is by $t$ test; count data is expressed by rate (\%), and comparison is by $\mathrm{X}^{2}$ test. The difference was statistically significant with $\mathrm{P}<0.05$.

\section{Result}

Intraoperative statistical results of 30 patients: the total bleeding volume of the patients was $34,900 \mathrm{ml}$, with an average bleeding volume of $(1163.33 \pm 678.86)$ $\mathrm{ml}$ per case; the total recovered blood volume was $17,997 \mathrm{ml}$, accounting for $51.6 \%$ of the total bleeding volume, and the average recovery per case was $(599.90 \pm 357.37) \mathrm{ml}$; Comparing the average bleeding volume with the reinfusion volume, $t=4.0226, P=0.0001$. During the operation, 12 patients were infused with plasma, a total of $5200 \mathrm{ml}$, with an average infusion of (433.33 \pm 166.97) $\mathrm{ml}$ per case, and $12 \mathrm{U}$ of concentrated red blood cells were infused. The patient's white blood cell (WBC), red blood cell (RBC), red blood cell pressure (HCT), hemoglobin (HGB), and coagulation time (PT) before and after autologous blood transfusion were compared, and the difference was statistically significant before and after surgery $(\mathrm{P}<0.001)$, While platelets $(\mathrm{PLT})$, activated prothrombin time (APTT) and fibrinogen (FIB) did not change significantly, the difference was not statistically significant $(\mathrm{P}>0.05)$. The specific results are shown in Table 2 and Table 3 below. All recovered washed red blood cells were

Table 2. Comparison of blood routine related indexes of 30 autotransfusion patients before and after surgery.

\begin{tabular}{ccccccc}
\hline & Cases & WBC & RBC & HCT & HGB & PLT \\
\hline Preoperative & 30 & $9.85 \pm 4.52$ & $4.51 \pm 0.63$ & $39.74 \pm 5.73$ & $127.95 \pm 21.23$ & $210.60 \pm 65.94$ \\
Postoperative & 30 & $13.85 \pm 4.78$ & $3.81 \pm 0.61$ & $32.98 \pm 4.57$ & $108.60 \pm 16.27$ & $209.37 \pm 70.74$ \\
t value & - & 3.3303 & 4.3722 & 5.0518 & 3.9624 & 0.0697 \\
P value & - & 0.0008 & 0.0000 & 0.0000 & 0.0001 & 0.4724 \\
\hline
\end{tabular}

Table 3. Comparison of Blood Coagulation Function related indexes of 30 autotransfusion patients before and after surgery.

\begin{tabular}{ccccc}
\hline & Cases & PT & APTT & FIB \\
\hline Preoperative & 30 & $12.12 \pm 1.18$ & $28.55 \pm 3.46$ & $3.57 \pm 1.32$ \\
Postoperative & 23 & $13.38 \pm 1.35$ & $27.47 \pm 3.16$ & $3.54 \pm 1.21$ \\
t value & - & 3.6192 & 1.1688 & 0.0850 \\
P value & - & 0.0003 & 0.1239 & 0.4663 \\
\hline
\end{tabular}


collected into blood transfusion bags by strict aseptic operation techniques, filtration, purification, washing and so on, and then returned to the patients during the operation. One of the 30 patients had a rash, and the rest recovered smoothly without any adverse reactions to blood transfusion.

\section{Discussion}

Autologous blood transfusion is an important method of blood transfusion in surgery in order to deal with the increasing tension of blood source. In our country, with the advent of aging, social development and scientific and technological progress, people's demand for health and improving the quality of life is increasing. Therefore, surgery improves the quality of life of patients, resulting in an increase in the amount of surgery and the amount of clinical blood used. Especially under the influence of the current new coronavirus epidemic, the blood source is even more tense, and the rare blood type is severely lacking, making it impossible for some rare blood type patients to perform surgery [9]. In addition, due to the impact of the new coronavirus epidemic, some family members of patients cannot go out to help each other donate blood, and some family members with blood diseases cannot donate blood, especially patients undergoing emergency surgery, which seriously affects the lives and treatment capabilities of patients; In the past, due to the window period of certain viral infections or the inability to detect the presence of the virus due to the level of technology, viral infections caused by allogeneic blood transfusion (such as hepatitis B, hepatitis C, HIV, etc.), immunosuppressive reactions, acute lung injury, circulation Potential complications such as overload and adverse reactions which cause people to panic [9] [10]. Based on the above reasons, many patients are unwilling to accept allogeneic blood transfusion in clinical work. With the application and development of autologous blood reinfusion technology, its advantages of simplicity, safety and effectiveness have been accepted by most patients.

In 2017, our hospital introduced the continuous autologous blood reinfusion system of Haemonetice Corporation from the United States; During the epidemic, 30 cases of spinal surgery patients had more bleeding during the operation; Autologous blood reinfusion combined with controlled blood pressure technology was used to collect the concentration of red blood cells from the blood of the patients with negative pressure aspirator through filtration, purification, and washing; The blood transfusion bag, which was returned to the patient during the operation and relieved the blood supply tension of the current new crown virus epidemic, and protected the life and health of the patient. During the operation, 13 cases were transfused with frozen plasma due to heavy bleeding, and 6 cases were transfused with allogeneic blood and frozen plasma due to excessive bleeding due to autologous blood transfusion, which could not meet the blood transfusion requirements, which greatly saved the blood source. The blood safety is guaranteed. 


\subsection{Autologous Blood Transfusion Technology Has its Indications and Contraindications [10]}

Indications: 1) Elective surgery with expected large intraoperative blood loss: cardiovascular surgery, orthopedic surgery, organ transplantation, etc.; 2) Patients with large blood vessels in the head and neck, spinal arteries and veins, and pelvic fractures; 3) Blood matching Difficulties, allogeneic blood transfusion and severe complications of blood transfusion. Contraindications: 1) Patients with bone malignant tumors and bone and joint tuberculosis; 2) Patients with bone infection, bacteremia, and sepsis.

\subsection{Advantages of Autologous Blood Transfusion}

1) Autologous blood transfusion solves the needs of family members unable to donate blood to each other and family members suffering from blood diseases under the new crown virus epidemic. 2) Effectively reduces blood transfusion costs and facilitates the implementation of DRG payments. 3) Autologous blood transfusion can not only save the blood source, but also relieve the patient's fear of various complications such as diseases caused by virus infections caused by allogeneic blood transfusion, and avoid disputes caused by diseases transmitted by allogeneic blood transfusion. 4) Avoid cases of special blood (such as $\mathrm{R} \mathrm{h}$ negative) that cannot be operated due to lack of blood supply. 5) At the same time, the vitality of autologous red blood cells is better than bank blood. Nie Sang, Miao Yuliang, etc. [11] [12] clinical studies have shown that autologous blood transfusion can reduce patient costs and reduce complications such as virus infection due to allogeneic blood transfusion.

\subsection{Complications of Autologous Blood Transfusion}

1) Like conventional blood transfusion, complications such as fever, hemoglobinuria, allergic reactions, and coagulation abnormalities also occur in autologous blood transfusion; 2) Bleeding complications: the blood endogenous heparin concentration of normal people is very low, and proper heparin concentration during surgery is a prerequisite for blood recovery which too low cannot achieve the purpose of anticoagulation, and too high can easily cause spontaneous bleeding; Therefore, a proper and reasonable heparin concentration is of great significance to the success or failure of the operation. 4) Coagulation dysfunction. Chen Lingkun, Wang Weiming et al. [13] [14] study that patients with massive bleeding (more than $3000 \mathrm{ml}$ ) lose too much platelets and coagulation factors in the blood, so long as the coagulation factors in the body maintain part of the coagulation function. Therefore, patients with massive bleeding need to be infused with fresh frozen plasma, which contains suspected blood factor components and can supplement the clotting factor components lost due to massive blood loss. Chen Lingkun, Wang Weiming et al. [13] [14] study that patients with massive bleeding (more than $3000 \mathrm{ml}$ ) lose too much platelets and coagulation factors in the blood, so long as the coagulation factors in the body maintain 
part of the coagulation function. Therefore, patients with massive bleeding need to be infused with fresh frozen plasma, which contains suspected blood factor components and can supplement the clotting factor components lost due to massive blood loss. Liu Lin et al. [15] studied the coagulation function of preschool children undergoing spinal orthopedic surgery who also confirmed this point. Patients with small and medium bleeding will not cause coagulation dysfunction, and generally do not need to infusion of fresh frozen plasma.

\section{Conclusions}

This study included 30 cases of spine surgery patients admitted from December 2019 to June 2020 during the epidemic. During the operation and after the operation, the Haemonetice Corporation autologous blood reinfusion system of the United States was used to return the blood recovered during the operation to the patient. It can effectively alleviate the shortage of blood sources under the influence of the current new crown virus epidemic, and protect the lives of the people, achieve double social and economic benefits, and solve the problem of patients with the new crown virus epidemic making full use of autologous blood transfusion and reducing allogeneic blood transfusion. When the reaction occurs, the amount of allogeneic blood transfusion is greatly reduced, medical expenses are reduced, and the application prospect is broad. This technology is worthy of promotion, especially in primary hospitals, which is of great significance [16] [17] [18].

In this study, the comparison of white blood cell (WBC), red blood cell (RBC), red blood cell pressure (HCT), hemoglobin (HGB) and clotting time (PT) of patients before and after autologous blood transfusion showed statistically significant differences before and after operation $(\mathrm{P}<0.001)$; however, platelets (PLT), activated prothrombin time (APTT) and fibrinogen (FIB) did not change significantly, and the difference was not statistically significant $(\mathrm{P}>0.05)$; Although there were certain differences in the relevant blood routine indexes and coagulation function indexes before and after autotransfusion, the indexes in all cases were still within the normal acceptable range, so there was no need for related treatment.

To sum up, during the COVID-19 pandemic, due to more blood loss during spinal surgery than other surgeries, spinal internal fixation surgery used a recovery machine to collect intraoperative blood for reinfusion. During the operation, blood pressure was controlled by antihypertensive drugs to a certain degree. The effect of postoperative clinical observation was significant and safe, especially during the current epidemic period.

\section{Limitations of the Study}

This study mainly observes a limited number of cases, which has certain limitations, and the number of cases can be expanded to obtain more powerful evidence. In addition, the follow-up time of this study is relatively long, and the pa- 
tient's compliance with follow-up has a certain complexity, which has a certain impact on the results.

\section{Acknowledgements}

During the process of this topic research, we got much help from many departments and individuals, and other personnel not involved in this project research. All of them offered a great support and help in this research. Now here, all of members in this research show our deepest appreciation to them, and wish them good health and everything goes well.

\section{Fund Project}

National Natural Science Foundation of China, contract project number: 71563056; Baise City Scientific Research and Technology Development Plan Project, project assignment number: 20182518.

\section{Conflicts of Interest}

The authors declare no conflicts of interest regarding the publication of this paper.

\section{References}

[1] Demetriades, D., Kimbrell, B., Salim, A., et al. (2005) Trauma Deaths in a Mature Urban Trauma System: Is "Trimodal" Distribution a Valid Concept? Journal of the American College of Surgeons, 201, 343-348.

https://doi.org/10.1016/j.jamcollsurg.2005.05.003

[2] Wessels, L.E., Calvo, R.Y., Sise, M.J., Bowie, J.M., Butler, W.J., Bansal, V. and Sise, C.B. (2020) Association of Operative Repair Type and Trauma Center Designation with Outcomes in Ruptured Abdominal Aortic Aneurysm Repair. Vascular and Endovascular Surgery, 54, 325-332. https://doi.org/10.1177/1538574420907193

[3] Kornas, R.L., Smith, S.W., Fagerstrom, E., Hendrickson, A., Tersteeg, J., Plummer, D., Driver, B.E. and Strobel, A.M. (2020) Spectrum and Frequency of Critical Procedures Performed at a Level I Adult and Pediatric Trauma Center. American Journal of Emergency Medicine. https://doi.org/10.1016/j.ajem.2020.04.002

[4] Han, C.X., Wang, J. and Ge, Y.L. (2019) Correlative Analysis of Hidden Blood Loss after Total Hip Replacement and Total Knee Replacement in the Elderly. PLA Preventive Medicine Journal, 37, 110-111.

[5] Wu, W., Wu, M.L., Hou, Z.Y., Jiang, J.Y., Li, B., Gao, Y.Z., Shen, H.Y., Luo, Z.J., Zhang, Y.Z., Guo, F.J., You, H.B., Xiao, J., Luo, Z.Q., Kang, H., Xiong, W., Zhu, W.T., Fang, Z., Liao, H., Chen, A. and Li, F. (2020) Expert Consensus on the Standardized Process of Orthopedics Diagnosis and Treatment during the Novel Coronavirus Pneumonia Epidemic Period. Bio-Orthopedic Materials and Clinical Research, 17, 1-7.

[6] Zhang, Y.S., Hai, Y., Zhang, Q., Zhao, C.S., Zhang, Y., Su, Q.J., Yang, J.C. and Sun, S. (2020) Protection Strategies for Orthopedic Surgery under the New Coronavirus Pneumonia Epidemic. Chinese Journal of Orthopaedics, 28, 289-292.

[7] Lu, H.Z., Cui, Y., Wang, J., Li, D.Z., Zhu, Y.B., Yin, Y.C. and Zhang, Y.Z. (2020) Analysis of Influencing Factors of Changes in Fracture Site of Orthopedic Hospita- 
lized Patients during the New Coronavirus Pneumonia Epidemic in Shijiazhuang Area. Chinese Journal of Trauma, No. 3, 202-206.

[8] Wang, X.L., Li, L., He, T.Y., Li, Z., Wu, C., Zhu, Y.B. and Zhang, Y.Z. (2020) Retrospective Analysis of Anesthesia Options for Orthopedic Trauma Surgery in the New Coronavirus Pneumonia Epidemic. Chinese Journal of Traumatology and Orthopedics, No. 5, 405-410.

[9] Luo, M.L., Yang, J. and Zheng, X.P. (2019) Autologous Blood Transfusion Can Improve Blood Oxygenation and Reduce Inflammatory Response in Orthopedic Surgery Patients. Genomics and Applied Biology, 38, 1741-1746.

[10] Xu, X.K., Lu, Z.Y. and Song, N.S. (2019) Application Observation of Patients' Autologous Blood Reinfusion in Spinal Surgery. Electronic Journal of Clinical Medicine, 6, 58-59.

[11] Nie, S., Li, L. and Cai, H.W. (2019) Retrospective Analysis of 71 Cases of Pediatric Orthopedic Patients Using Autologous Blood Transfusion. Chinese Medical Engineering, 27, 68-72.

[12] Miao, Y.L., Liu, Y., Wang, X.P., Ma, H.S., Fang, W.W. and Shi, W.Z. (2019) The Effect of Autologous Blood Retransfusion during Scoliosis Correction Surgery in School-Age Children on Blood Saving and Cost. Zhejiang Medicine, 41, 810-812+816.

[13] Chen, L.K., Su, W. and Zhang, M. (2019) The Effect of Different Concentrations of Heparin in Washing Autologous Blood Reinfusion on the Coagulation Function of Surgical Patients. Thrombosis and Hemostasis, 25, 112-114.

[14] Wang, W.M. (2018) The Effect of Intraoperative Autologous Blood Transfusion on Coagulation Function in Patients with Spinal Surgery. Chinese Medical Innovation, 15, 110-113.

[15] Liu, L., Zhang, J.M., Zhao, X., Ren, Y., Fu, W.Y. and Feng, L. (2019) Study on the Effect of Intraoperative Recovery Autotransfusion on Coagulation Function of Preschool Children Undergoing Spinal Orthopedic Surgery. Journal of Clinical Pediatric Surgery, 18, 579-582.

[16] Huang, S.Z., Zhong, H.J., Chen, L.H., Chen, W.Z., Yu, M. and Chen, W.S. (2019) Intraoperative Autotransfusion Technique in Spinal Surgery. Chinese National Health and Medical Sciences, 31, 62-64.

[17] Guo, F., Ge, Y.L. and Chen, X.P. (2018) The Effect of Preoperative Autotransfusion Combined with Intraoperative Autotransfusion in Complex Spinal Surgery. Clinical Transfusion and Laboratory Medicine, 20, 380-384.

[18] Shen, H.W., Zhang, P., Zhong, Z.H. and Zhou, W. (2018) Study on the Effectiveness of Different Methods of Autologous Blood Transfusion during the Perioperative Period of Lumbar Interbody Fusion in Elderly Patients. Journal of North Sichuan Medical College, 33, 202-205. 